\title{
Factors influencing the need for recovery in employees with hearing loss: a cross-sectional study of health administrative data
}

\author{
Hanneke E. M. van der Hoek-Snieders ${ }^{1}$ [D $\cdot$ Monique Boymans $^{1} \cdot$ Bas Sorgdrager $^{2} \cdot$ Wouter A. Dreschler $^{1}$
}

Received: 25 September 2019 / Accepted: 28 May 2020 / Published online: 7 June 2020

(c) The Author(s) 2020

\begin{abstract}
Objective Need for recovery is a predictor of work stress and health problems, but its underlying factors are not yet well understood. We aimed to identify hearing-related, work-related, and personal factors influencing need for recovery in hearingimpaired employees.

Methods We retrospectively identified hearing-impaired employees $(N=294)$ that were referred to the Amsterdam University Medical Center between 2004 and 2019. Routinely obtained healthcare data were used, including a survey and hearing assessments. A directed acyclic graph was constructed, revealing the hypothesized structure of factors influencing need for recovery as well as the minimal set of factors needed for multiple regression analysis.

Results Four variables were included in the regression analysis. In total, $46.1 \%$ of the variance in need for recovery was explained by the factors feeling that something should change at work $(B=19.01, p<0.001)$, self-perceived listening effort $(B=1.84, p<0.001)$, personal adaptations scale score $(B=-0.34, p<.001)$, and having a moderate/poor general health condition $(B=20.06, p<0.001)$. Although degree of hearing loss was associated with self-perceived listening effort, the direct association between degree of hearing loss and need for recovery was not significant.

Conclusions The results suggest that the way employees perceive their hearing loss and how they cope with it directly influence need for recovery, rather than their measured degree of hearing loss. Additionally, general health condition was found to be an independent factor for need for recovery. The results should be confirmed by future, longitudinal research.
\end{abstract}

Keywords Workers $\cdot$ Fatigue $\cdot$ Hearing loss $\cdot$ Listening effort $\cdot$ Work participation $\cdot$ Communication strategies

\section{Introduction}

Hearing loss is a common occupational malady (Backenroth-Ohsako et al. 2003; May 2000). Prevalence estimates vary from 7 to $31 \%$ and increase with age and exposure to noise (Hasson et al. 2010; Masterson et al. 2016; May 2000; Nelson et al. 2005). It is estimated that $3 \%$ of the Dutch work force experiences difficulties in their job due to their hearing loss (Sorgdrager 2015). These difficulties often result in greater levels of fatigue, fear, social isolation,

Hanneke E. M. van der Hoek-Snieders

h.e.vanderhoek@amsterdamumc.nl

1 Amsterdam UMC, Department of Clinical and Experimental Audiology, University of Amsterdam, Meibergdreef 9, 1105 AZ Amsterdam, The Netherlands

2 Amsterdam UMC, University of Amsterdam, Netherlands Centre of Occupational Diseases, Coronel Institute AmCOGG, Meibergdreef 9, Amsterdam, The Netherlands and psychophysiological stress, caused by the fact that hearing loss goes along with increased listening effort during activities, such as communicating in background noise or localizing sounds (Hornsby and Kipp 2016; Kramer et al. 2006; Morata et al. 2005; Ohlenforst et al. 2017; Svinndal et al. 2018). Adverse implications for work are sick leave due to mental distress, unemployment, and earlier retirement (Danermark and Gellerstedt 2004; Hasson et al. 2011; Kramer et al. 2006; Punch 2016). The degree of hearing loss is significantly associated with need for recovery (NFR) (Nachtegaal et al. 2009), a measure that can contribute to early identification of occupational diseases (de Croon et al. 2003; Moriguchi et al. 2010; Sluiter et al. 2003; Sluiter 1999).

NFR has been defined as the need to recuperate from work induced fatigue, primarily experienced after a day of work (Jansen et al. 2002; van Veldhoven and Broersen 2003). The degree of NFR is determined by the intensity of mental and physical work-induced fatigue and by the period 
required to return to a normal level of functioning. NFR can be measured with the validated Questionnaire on the Experience and Evaluation of Work (QEEW), which includes 11 dichotomous statements, such as 'I find it hard to relax at the end of a working day' and 'When I get home, people should leave me alone for some time' (van Veldhoven and Broersen 2003). NFR is a predictor of work stress, subjective health problems, and sick leave (de Croon et al. 2003; Sluiter et al. 2003). In line with the International Classification of Functioning, Disability and Health (ICF) (World Health Organization, 2001). NFR has been described to be a complex construct that is influenced by disease specific, personal, and environmental factors (Gommans et al. 2015).

Despite the importance of the outcome NFR both from health and economic perspectives, the studies examining NFR in patients with hearing loss are scarce. To our knowledge, three studies have been reported so far. In the crosssectional study by Nachtegaal et al. (2009), the relationship between NFR and hearing status was examined in 925 normally hearing and hearing-impaired working adults. NFR was assessed with the QEEW and hearing status with the national hearing test (Smits et al. 2006), a speech-in-noise test that was performed over the internet. Their regression analysis showed that poorer hearing was significantly associated with higher need for recovery. In the cross-sectional study by Juul Jensen et al. (2018), the relationship between NFR and tinnitus was examined in 32 hearing aid users of which 16 were suffering from tinnitus. NFR was assessed with a Danish translation of the QEEW and tinnitus with the Tinnitus Handicap Inventory. The authors reported that the degree of tinnitus severity was significantly associated with higher NFR. Finally, a randomized controlled trial has been reported by Gussenhoven et al. (2017) in a population of 136 hearing-impaired employees. The study evaluated the effectiveness of a vocational enablement protocol on NFR as compared to usual care for hearing-impaired employees. This protocol is a multidisciplinary program of care that consists of vocational and audiological components, such as an intake interview conducted by the psychologist or social worker and clinical occupational physician, the performance of tone audiometry and a speech-in-noise test, and a multidisciplinary team meeting in which the technical, speech therapeutic, and psychosocial intervention options are discussed (Gussenhoven et al. 2012). The intervention of the control group consisted of any kind of another audiological revalidation. NFR had not significantly changed after 12 months follow-up, and there were no significant differences between the intervention and the control group (Gussenhoven et al. 2017). The authors concluded that NFR may not adequately capture what is covered in the vocational enablement protocol. However, it is unclear how many employees received technical, speech therapeutic, and psychosocial interventions and thus which interventions did not influence
NFR. Further, because the factors influencing NFR in hearing-impaired employees are not yet well understood, it is difficult to indicate which changes in degree of hearing loss could have an effect on NFR.

Multiple studies have indicated work characteristics influencing NFR, such as the number of working hours (Jansen et al. 2002; Verdonk et al. 2010), lack of participation in work decisions (van Veldhoven and Broersen 2003), and problems in the relationship with colleagues (Kiss et al. 2008; van Veldhoven and Broersen 2003). High job demands and low job support are associated with high NFR and mixed results are presented for job control (Kiss et al. 2008; Kraaijeveld et al. 2014; Sluiter et al. 2001; Sonnentag and Zijlstra 2006; Van der Hulst et al. 2006). Job demands and job control have also been demonstrated to be associated with NFR in hearing-impaired employees, independently of the degree of hearing loss measured with an online hearing test (Nachtegaal et al. 2009). To our knowledge, further studies examining the effect of work characteristics on NFR in hearing-impaired employees are lacking, but high auditory job demands were shown to be related to sick leave due to stress-related complaints (Kramer et al. 2006).

Personal characteristics influencing NFR in the general working population include gender (Kiss et al. 2008), age (Gommans et al. 2015; Kiss et al. 2008), general health condition (Gommans et al. 2015; van der Starre et al. 2013), educational level (de Croon et al. 2003), and coping style (de Vries et al. 2015; Machin and Hoare 2008). Several studies have indicated that people with hearing loss use coping strategies in their interaction with others (Backenroth-Ohsako et al. 2003; Barker et al. 2017; Hallberg and Carlsson 1991). Also, the Communication Profile for the Hearing Impaired (CPHI) has been developed to investigate how people cope with their hearing loss (Mokkink et al. 2009). This questionnaire contains questions on the communication strategies and non-verbal strategies that are commonly used by people with hearing loss. However, the influence of hearing loss coping on NFR has not yet been examined.

The evidence on factors influencing NFR in hearingimpaired employees lags behind, although the outcome NFR has potential for early identification of hearing-impaired employees being at risk for occupational diseases, and may be a valuable tool for evaluating the effects of interventions aiming to prevent these problems. It is hypothesized that hearing loss, work characteristics, and personal characteristics influence both each other and NFR. Because earlier studies do not provide a framework on how these factors interfere, NFR may not be optimally understood in employees with hearing loss. The primary aim of this study is therefore to identify hearing-related, work-related, and personal factors influencing NFR in hearing-impaired employees. To examine if the influence of hearing-related, work-related, and personal factors on NFR differs from their influence on 
listening effort, which is a more commonly assessed construct when assessing the functional disability of hearingimpaired employees, the secondary aim is to identify factors influencing listening effort.

\section{Methods}

\section{Design}

We performed a single center study with an observational and cross-sectional design at the Amsterdam University Medical Center (Amsterdam UMC). Factors potentially influencing NFR were derived from hearing assessments performed at the hospital and a hearing survey that was completed at home. All variables were derived from patient files.

\section{Participants}

We retrospectively identified patients referred to Amsterdam UMC's ENT-Audiology department (location AMC) by their occupational physician. All patients were thus referred from occupational healthcare. Eligible patients visited the hospital between 2004 and 2019, were aged between 18 and 67 , underwent pure tone and speech audiometry, and completed the hearing survey prior to their hospital visit. Patients were included regardless of the cause of their hearing loss. For patients with multiple referrals, the data were included belonging to the first referral with a completed questionnaire and hearing evaluation. To prevent bias, patients were excluded if they were referred to the hospital for a fitness for job assessment by their employer. The reason is that hearing loss complaints might be experienced or reported differently if continuation of the job depends on it. All patients received a letter with information about the study. Because of the retrospective study design, an opt-out procedure was performed.

A total of 646 patients were identified of being referred to the ENT-Audiology department by their occupational physician (Fig. 1). Patients referred to the department to determine fitness for their job $(n=283)$ were not eligible for the study, as were patients older than $67(n=2)$. Further, patients were excluded that declined to participate $(n=6)$ or had an incomplete patient file, specifically missing tone audiometry $(n=4)$, missing survey $(n=20)$, or incomplete survey $(n=37)$.

\section{Data collection}

The data were collected retrospectively by review of patient files. Outcomes of the hearing survey and the hearing assessments were entered into Castor, an electronic database (Castor EDC 2019) by the author and a research assistant. Data entry was checked in a sample of $50 \%$ of the cases. Information not available in patient files were noted as missing.

\section{Hearing assessment}

The hearing assessments consisted of unaided pure tone and speech audiometry for all patients. At the ENT-Audiology Department, speech reception tests in noise are not routinely performed in all patients, but only if understanding speech in a noisy environment is important for job performance. Therefore, speech reception tests in noise were performed depending on the profession and the associated auditory demands.

Fig. 1 Flow chart

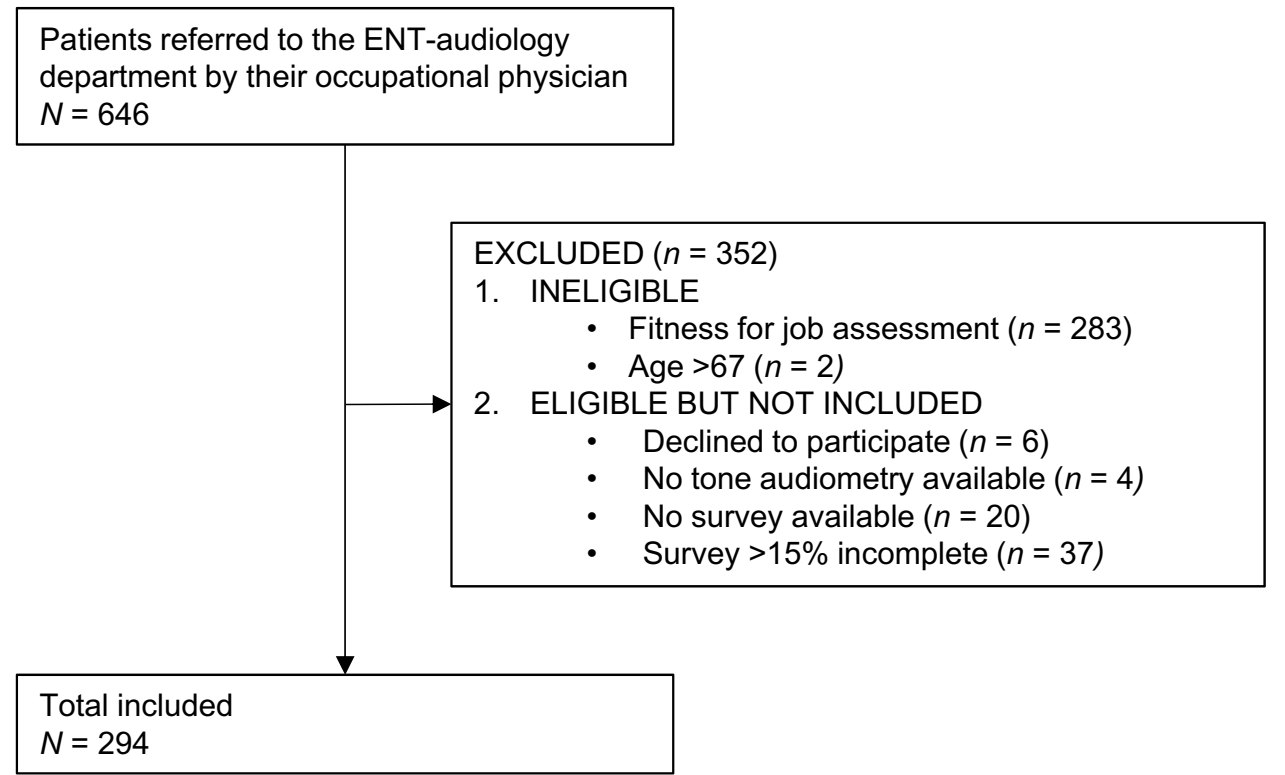




\section{Pure tone and speech audiometry}

Pure tone and speech audiometry (ISO 8253-1, 1989) were performed in a sound-isolated booth using calibrated clinical audiometers (AC40 and Decos audioNigma) and TDH 39 headphones. According to the hospital protocol, pure tone thresholds for air and bone conduction were reported in decibel (dB) hearing level (HL) at frequencies $250 \mathrm{~Hz}, 500 \mathrm{~Hz}, 1000 \mathrm{~Hz}, 2000 \mathrm{~Hz}, 4000 \mathrm{~Hz}$, and $8000 \mathrm{~Hz}$, with adequate masking (if necessary). For a healthy, normally hearing individual, hearing thresholds up to $25 \mathrm{~dB}$ HL are regarded as normal (Martin and Champlin 2000). According to the American Academy of Otolaryngology, Binaural Hearing Impairment (BHI) was calculated from the mean of pure tone thresholds for air conduction at $1000 \mathrm{~Hz}, 2000 \mathrm{~Hz}$, and $4000 \mathrm{~Hz}$ and a 5:1 weighting favoring the better ear (American Academy of Otolaryngology 1979). BHI provides a valid estimation of hearing disability that a person with a degree of hearing loss would experience (Dobie 2011). Speech audiometry was performed with the standard Dutch CVC word lists (Bosman and Smoorenburg 1995) and was used to calculate the percentage of maximum speech recognition for the better ear. It has been recommended to transform percentages of maximum speech recognition, to enhance the normality of the data for the statistical analysis (Sherbecoe and Studebaker 2004). Therefore, we transformed the percentages of maximum speech recognition using the rationalized arcsine formula (Sherbecoe and Studebaker 2004).

\section{Speech reception threshold test}

Speech reception in continuous noise was assessed in a free field setting (SON0) using everyday Dutch sentences developed by Plomp and Mimpen (1979) or the sentences developed by Versfeld et al. (2000). These sentence materials have been shown to result in similar test outcomes (Versfeld et al. 2000). The aim of performing a speech reception test in noise is to objectify the influence of the hearing loss on functional speech understanding at the workplace. Therefore, all patients were asked if they wear hearing aids at work. If they did, the critical Signal to Noise Ratio (SNR) was measured for aided conditions. If they did not, the SNR was measured for the unaided conditions. We have combined the SNR-outcomes of patients who performed the test with and without hearing aids. We will refer to these measurements as speech in noise tests performed in patient's daily work situation. For a healthy, normally hearing individual, an SNR below -4 can be expected (Versfeld et al. 2000).

\section{Survey}

Prior to the hospital visit, patients completed an extensive hearing survey to investigate personal and environmental factors that potentially influence NFR. This survey consisted of three questionnaires and additional questions.

\section{Experience and evaluation of work}

Three scale scores were derived from the QEEW, a generic questionnaire on psychosocial workload and work stress (Van Veldhoven et al. 2002). The sum score of each QEEW scale can be converted to a scale score ranging from 0 to 100. A higher score represents a higher level of the working condition. First, the primary outcome was operationalized with the NFR-scale score that is composed of 11 items, such as "I find it hard to relax at the end of a working day". An NFR-scale score higher than 54 indicates an increased risk for occupational and health problems (Broersen et al. 2004). Second, the score on the scale participation at work was included as a work-related factor. This scale consists of 8 items, such as "Can you participate in decisions about the nature of your work?". Third, the score on the scale collegial support was included as a work-related factor. This scale consists of 9 items, such as "If necessary, can you ask your colleagues for help?" The QEEW has been shown to be reliable with good internal consistency and multiple studies have concluded good validity (Van Veldhoven et al. 2002, 2015). For example, the NFR scale has been shown to have good content-, construct-, and criterion-related validity in relation to work related health (Van Veldhoven and Sluiter 2009).

\section{The Amsterdam checklist for hearing and work}

A 4-point response scale was used to inventory the occurrence of six hearing-related job activities, specifically detecting sounds, distinguishing sounds, communication in quiet, communication in noise, localizing sounds, and exposure to loud sounds. These questions on the occurrence of hearingrelated job activities were merged into a value representing auditory demands at work by calculating a weighted sum score. Communication in quiet and distinguishing sounds are considered to be the easiest hearing activities and received a weighting of 1 . Detecting and localizing sounds are considered to be of moderate difficulty and received a weighting of 2. Exposure to loud sounds and communication in noise are considered to be the most difficult and received a weighting of 3 . This score can range between 0 and 48 .

We did not only investigate the occurrence of the six hearing-related job activities, but also the effort they take. Since these six additional questions on the effort of hearing have good internal consistency $(\alpha=0.81)$, we have 
calculated a sum score of these six items, further considered as self-perceived listening effort. This score can range between 0 and 18 .

Other questions derived from the Amsterdam Checklist for Hearing and Work (ACHW) include the number of working hours a week (scale value) and fulfilling managerial tasks (dichotomous). All patients were asked whether they fulfilled managerial tasks, because managerial activities can be embedded in many professions and require specific skills that might appeal to hearing acuity, such as organizational and social skills (Whitley 1989).

\section{Communication Profile for the Hearing Impaired}

To investigate the coping strategies, the CPHI was used. The CPHI aims to distinguish between adequate and inadequate coping behavior (Mokkink et al. 2009). It has been translated and validated for Dutch and contains two domains. The first domain regards communication strategies and contains 8 items for maladaptive behavior, 8 items for verbal strategies, and 7 items for nonverbal strategies. For example, items within the domain of communication strategies are "I avoid conversations, because of my hearing loss" (maladaptive behavior), "When I don't understand what is being said, I ask for a repeat" (verbal strategies), and "I always try to watch a person's face" (non-verbal strategies). The second domain regards personal adjustments and contains 6 items for self-acceptance, 8 items for acceptance of loss, and 15 items for stress and withdrawal. For example, items within the domain of personal adjustments are "I get mad at myself when I can't understand others" (self-acceptance), "I can't talk to people about hearing loss" (acceptance of loss), and "I get tense, because of my hearing loss" (stress and withdrawal). The CPHI scales are scored such that low scores are indicative of communicative of adjustment difficulties.

\section{Additional survey questions}

Additional questions included the personal characteristics age, gender, educational level, and general health condition. For health condition, the response options were good, moderate, and bad. We used a dichotomous question to ask if the employees were feeling that something should change in their work situation. Two hearing-related characteristics were inventoried with a dichotomous question, specifically the presence of tinnitus, and the use of hearing aids.

\section{Variables in the analysis}

A total of 17 factors potentially influencing NFR were explored for eligibility in the statistical model (Table 1). The same factors were explored for the secondary analysis. Hearing-related factors consisted of self-perceived listening effort, BHI, the maximum speech discrimination score, the critical Signal-to-Noise Ratio (SNR) measured in the speech-in-noise test, and the presence of tinnitus. Workrelated factors consisted of work participation, collegial support, auditory demands at work, fulfilling managerial tasks, the number of working hours a week, and feeling that something should change at work. Personal factors consisted of the communication strategies and personal adjustments used, and general health condition. In addition, age, gender, and educational level were considered to be potential confounders.

\section{Statistical analysis}

Distributions of all variables were examined. For continues variables, the means and standard deviations were calculated and histograms were used to check normality. For categorical variables, proportions were calculated. We drew a directed acyclic graph to reduce the required sample size and prevent power issues without missing factors related to the outcome measure and without missing factors required to reduce bias. This method aims to assist in the selection of appropriate variables for the regression analysis, as is recommended by Greenland et al. (1999). Afterwards, multiple linear regression was performed.

\section{Directed acyclic graph}

We visualized our hypothesized relationships between the factors and their association with the primary outcome NFR and secondary outcome self-perceived listening effort. To simplify the graph, we examined the correlations between the factors in the graph and removed all negligible associations, defined as correlation coefficients between -0.3 and +0.3 (Hinkle et al. 2003). Pearson correlation coefficients were used to examine the correlations between continuous variables, the Phi correlation coefficients for dichotomous variables, and the Bi-serial correlation coefficients to determine the correlation between a dichotomous and a continuous variable (Akoglu 2018; Kraemer 2014). Further simplification was accomplished by following the method of Shrier \& Platt (2008), including removal of all factors that were not directly or indirectly related to neither the primary nor the secondary outcome.

\section{Multiple imputation}

Multiple imputation was used to impute factors directly or indirectly related to the primary or secondary outcome (Pedersen et al. 2017). The number of imputations was 10, thus 10 imputed datasets were created. The imputation model consisted of all variables included in the conceptual model (Table 1). 
Table 1 Factors hypothesized to influence on need for recovery

\begin{tabular}{|c|c|c|}
\hline & Derived from & Operationalization \\
\hline Binaural hearing impairment & Pure tone audiometry & $\begin{array}{l}\text { Sum of pure tone thresholds at } 1,2 \text {, and } 4 \mathrm{kHz} \text { with a } 5: 1 \text { weighting favoring the } \\
\text { better ear }\end{array}$ \\
\hline Maximum discrimination & Speech audiometry & Percentage of maximum speech recognition for the better ear \\
\hline SNR in continuous noise & Speech recognition test & SNR measured in the daily life situation at work (with or without hearing aids) \\
\hline Presence of tinnitus & Survey4 & Item score (dichotomous) \\
\hline Work participation & Survey (QEEW) & Standardized scale score \\
\hline Collegial support & Survey (QEEW) & Standardized scale score \\
\hline Self-perceived listening effort & Survey (ACHW) & $\begin{array}{l}\text { Sum score of } 6 \text { questions (4-point scale) on experienced listening effort during } \\
\text { hearing-related job activities }\end{array}$ \\
\hline Auditory work demands & Survey (ACHW) & $\begin{array}{l}\text { Weighted sum score of } 6 \text { questions (4-point scale) on the occurrence of hearing- } \\
\text { related job activities }\end{array}$ \\
\hline Fulfilling managerial tasks & Survey (ACHW) & Item score (dichotomous) \\
\hline Number of working hours & Survey (ACHW) & Item score (open question) \\
\hline Feeling something should change & Survey & Item score (dichotomous) \\
\hline Communication strategies & Survey (CPHI) & $\begin{array}{l}\text { Standardized scale score consisting of maladaptive behavior, verbal strategies, and } \\
\text { non-verbal strategies }\end{array}$ \\
\hline Personal adjustments & Survey (CPHI) & $\begin{array}{l}\text { Standardized scale score consisting of self-acceptance, acceptance of loss and } \\
\text { stress, and withdrawal }\end{array}$ \\
\hline Age & Survey & Item score (open question) \\
\hline Gender & Survey & Item score (dichotomous) \\
\hline General health condition & Survey & Item score (dichotomized) \\
\hline Educational level & Survey & Item score (6 categories) \\
\hline
\end{tabular}

SNR Signal to Noise Ratio, QEEW Questionnaire on the Experience and Evaluation of Work; ACHW Amsterdam Checklist for Hearing and Work (ACHW), CPHI Communication Profile for the Hearing Impaired

\section{Linear regression analysis}

Linear regression with a forward stepwise selection method $(\alpha=0.05)$ was manually performed with all variables directly related to NFR. As a result of the strategy used to select factors for the analysis, the model was unadjusted for other factors. We checked for interaction effects with the use of hearing aids or not with all variables in the analysis, because the relationship between objective and subjective factors might be different for employees that wear hearing aids. If an interaction term was not found to be significant $(p>0.05)$, it was removed. Data organization and statistical analysis were performed using the Statistical Package for Social Sciences (SPSS) version 25.0 (Armonk New York USA). The critical value of significance was 0.05 for all statistical analyses.

\section{Results}

\section{Participants}

A total of 294 patients, mean age 56 (SD 8.9), were included the study (Table 2). Patients reported being in good health $(60.2 \%)$, moderate/poor health $(39.1 \%)$, and poor health $(0.7 \%)$. Since $0.7 \%$ of the cases used the third category, this question was dichotomized for the statistical analysis. The mean BHI was $41.3 \mathrm{~dB}$ HL (SD 20.76). For the maximum speech discrimination score in quiet, the median was $100 \%$ (range 15\%-100\%). The mean critical SNR was $-2 \mathrm{~dB}$ (SD 4.4). Hearing aids were used by $58.5 \%$ of the patients.

All educational levels were represented. The most common professions were teacher (26.6\%), administrative job (19.4\%), doctor/nurse (10.2\%), and managerial jobs (9.2\%). Many patients with and without managerial jobs reported to fulfil managerial tasks (88.4\%). The mean number of working hours per week was 33.6 (SD 8.7). The mean score for NFR was 54.94 (SD 34.12). In 55.8\% of the participants, the NFR score was above 54, indicating an increased risk at occupational and health problems (Broersen et al. 2004). The mean self-perceived listening effort was 10.28 (SD 4.05). The CPHI resulted in a mean score of 79.79 (SD 15.84) for communication strategies and 97.31 (SD 26.23) for personal adjustments. A normal distribution was confirmed for all variables, except for the percentage of maximum speech recognition. Even after application of the rationalized arcsine transformation (Sherbecoe and Studebaker 2004), the variable remained skewed to the right. This variable was therefore not used in the analysis. 
Table 2 Characteristics of the included participants $(N=294)$

\begin{tabular}{|c|c|c|c|c|}
\hline & $\%$ & Mean (SD) & Min; $\max$ & Missing $n$ \\
\hline Age & & $50.9(8.9)$ & $19 ; 65$ & 0 \\
\hline Gender ( $\%$ male $)$ & 58.6 & & & 0 \\
\hline General health condition & & & & 2 \\
\hline Good & 60.2 & & & \\
\hline Moderate/poor & 39.1 & & & \\
\hline Degree of hearing loss (weighted) & & & & 0 \\
\hline Normal hearing $(<15 \mathrm{~dB}$ HL) & 22.9 & & & \\
\hline Mild (25-40 dB HL) & 28.0 & & & \\
\hline Moderate (40-60 dB HL) & 31.7 & & & \\
\hline Severe (60-80 dB HL) & 11.3 & & & \\
\hline Profound ( $>80 \mathrm{~dB} \mathrm{HL}$ ) & 6.1 & & & \\
\hline Binaural hearing impairment ${ }^{\mathrm{a}}$ & & $41.3(20.8)$ & $3.8 ; 110.8$ & 0 \\
\hline Maximum discrimination & & $94.5(12.9)$ & $15 ; 100$ & 1 \\
\hline SNR in continuous noise & & $-2.2(4.4)$ & $-9 ; 14.6$ & 158 \\
\hline Presence of tinnitus ( $\%$ yes) & 63.9 & & & 4 \\
\hline Hearing aids (\% yes) & 57.5 & & & 3 \\
\hline Educational level & & & & 5 \\
\hline Primary/lower vocational & 7.6 & & & \\
\hline General intermediate & 7.6 & & & \\
\hline Intermediate vocational & 22.5 & & & \\
\hline General secondary & 10.0 & & & \\
\hline Higher vocational & 36.0 & & & \\
\hline University & 16.3 & & & \\
\hline Profession & & & & 1 \\
\hline Teacher & 26.6 & & & \\
\hline Administrative & 19.5 & & & \\
\hline Doctor/nurse & 10.2 & & & \\
\hline Manager & 9.2 & & & \\
\hline Coach/social worker & 5.5 & & & \\
\hline Construction worker & 4.4 & & & \\
\hline Police officer/fireman & 3.4 & & & \\
\hline Other & 21.2 & & & \\
\hline Number of working hours & & $33.6(8.7)$ & $16 ; 48$ & 1 \\
\hline Fulfilling managerial tasks (\% yes) & 88.4 & & & 21 \\
\hline Need for recovery (range $0-100$ ) & & $54.9(34.1)$ & $0 ; 100$ & 12 \\
\hline Work participation (range $0-100$ ) & & $49.0(22.8)$ & $0 ; 95.83$ & 11 \\
\hline Collegial support (range 0-100) & & $20.8(13.2)$ & $0 ; 55.56$ & 14 \\
\hline Self-perceived listening effort (range $0-18$ ) & & $10.3(4.1)$ & $0 ; 18$ & 20 \\
\hline Auditory demands at work (range $0-48$ ) & & $30.6(6.1)$ & $16 ; 48$ & 10 \\
\hline Feeling something should change ( $\%$ yes) & 45.2 & & & 24 \\
\hline Communication strategies (range 23-115) & & $79.8(15.8)$ & $0 ; 115$ & 7 \\
\hline Personal adjustments (range 29-145) & & $97.3(26.2)$ & $0 ; 145$ & 7 \\
\hline
\end{tabular}

SNR Signal to Noise ratio

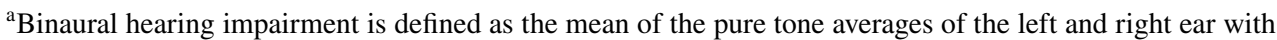
a 5:1 weighting favoring the better ear

\section{Directed acyclic graph}

The presence of tinnitus, age, and the educational level were not directly or indirectly associated with NFR and self-perceived listening effort. Consequently, these factors were not included in the directed acyclic graph. Figure 2 shows the directed acyclic graph that was constructed. Four variables directly influenced the primary outcome NFR, 


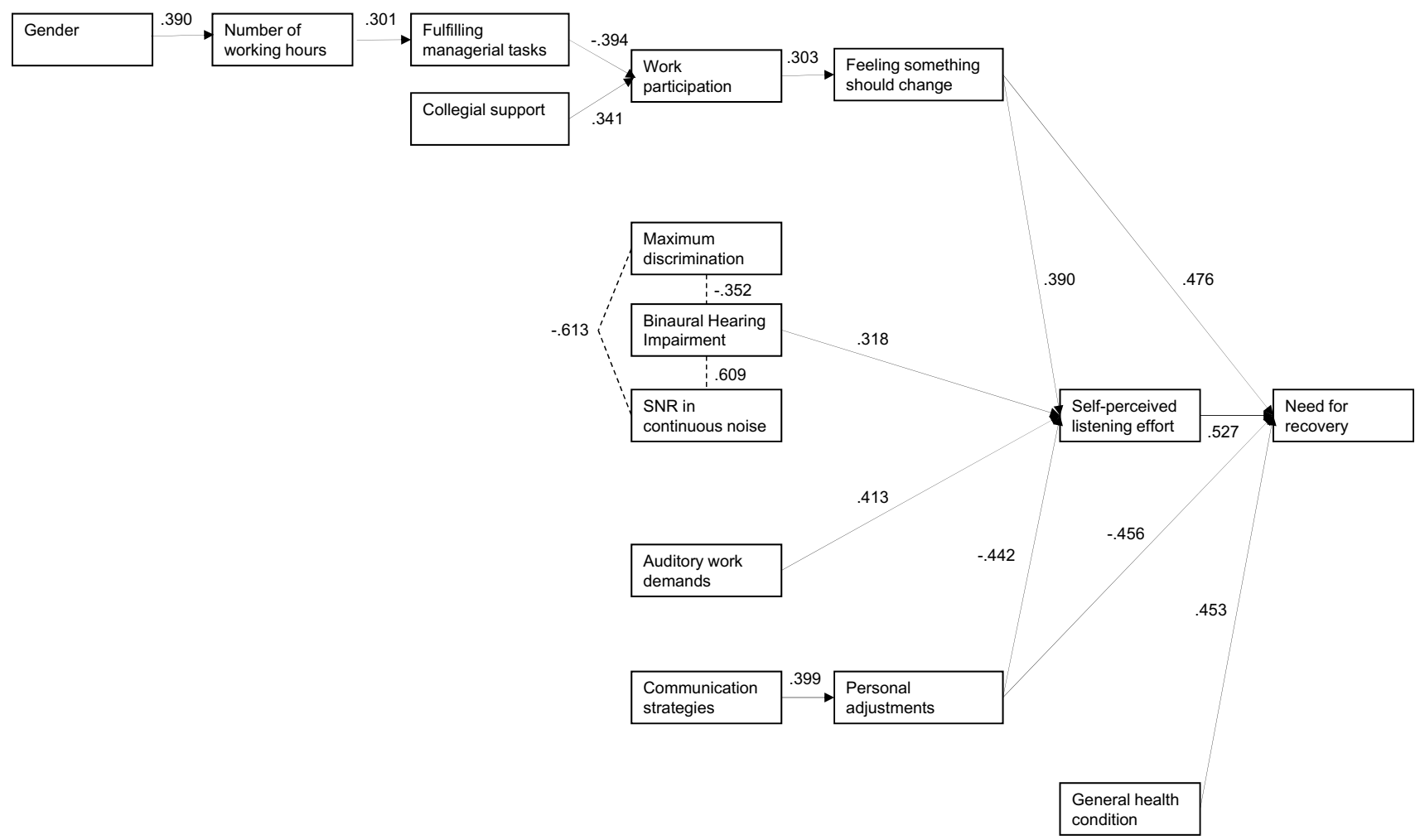

Fig. 2 Directed acyclic graph: a visualization of our hypothesized relationships between the factors and need for recovery that was simplified by removing all correlations between -0.3 and +0.3 , followed

specifically feeling that something should change at work $(r=0.476)$, self-perceived listening effort $(r=0.527)$, personal adaptations $(r=-0.456)$, and general health condition $(r=0.453)$. Two of these variables did also directly influence the secondary outcome measure self-perceived listening effort, respectively, feeling that something should change at work $(r=0.390)$, and personal adaptations $(r=-0.442)$. Self-perceived listening effort was also directly influenced by BHI $(r=0.318)$ and auditory work demands $(r=0.413)$. The hearing assessment outcomes did not significantly correlate with NFR, including BHI $(r=0.096, p=0.109)$, maximum discrimination score $(r=0.010, p=0.873)$, and SNR $(r=0.060, p=0.492)$. All correlations between the hypothesized factors and the primary and secondary outcome are shown in Table 3.

\section{Multiple regression analysis}

The results of the primary regression analysis (Table 4) indicated the four predictors explained $46.1 \%$ of the variance $(p<0.001)$ of the NFR. Feeling that something should change at work, self-perceived listening effort, the personal adaptations scale score, and having a moderate/poor general health condition were significantly related to higher NFR. by simplification according to the method of Shrier and Platt (2008). SNR Signal-to-Noise Ratio

Table 3 Correlations between the hypothesized factors and the primary outcome need for recovery and the secondary outcome self-perceived listening effort

\begin{tabular}{lcc}
\hline & Need for recovery & $\begin{array}{c}\text { Self-perceived } \\
\text { listening effort }\end{array}$ \\
\hline Binaural hearing impairment & 0.099 & 0.318 \\
Maximum discrimination & -0.024 & -0.167 \\
SNR in continuous noise & 0.060 & 0.203 \\
Presence of tinnitus & 0.102 & 0.094 \\
Work participation & 0.154 & 0.006 \\
Collegial support & 0.198 & 0.130 \\
Self-perceived listening effort & 0.527 & - \\
Auditory work demands & 0.226 & 0.413 \\
Fulfilling managerial tasks & -0.050 & 0.109 \\
Number of working hours & -0.152 & -0.071 \\
Feeling something should & 0.476 & 0.390 \\
change & & 0.197 \\
Communication strategies & 0.032 & -0.442 \\
Personal adjustments & -0.456 & 0.133 \\
Age & -0.018 & -0.133 \\
Gender & -0.186 & 0.289 \\
General health condition & 0.453 & 0.124 \\
Educational level & 0.108 & \\
\hline
\end{tabular}

SNR Signal to Noise Ratio 
Table 4 Results of multiple linear regression analysis of factors associated with need for recovery

\begin{tabular}{llrlr}
\hline & Variable & \multicolumn{1}{c}{$B$} & $95 \%$ CI & $p$ \\
\hline $\begin{array}{l}\text { Complete case } \\
\text { analysis }\end{array}$ & Constant & 32.03 & $9.12 ; 54.93$ & .006 \\
$R^{2}=0.495$ & Feeling something should change* & 19.01 & $12.04 ; 25.97$ & $<.001$ \\
& Self-perceived listening effort & 1.84 & $0.88 ; 2.81$ & $<.001$ \\
& Personal adaptations & -0.34 & $-0.49 ;-0.19$ & $<.001$ \\
& General health condition** & 20.06 & $13.18 ; 26.94$ & $<.001$ \\
Pooled analysis & Constant & 31.78 & $8.70 ; 54.86$ & .010 \\
after imputation & Feeling something should change* & 17.88 & $10.48 ; 25.29$ & $<.001$ \\
$R^{2}=0.461$ & Self-perceived listening effort & 1.93 & $0.97 ; 2.88$ & $<.001$ \\
& Personal adaptations & -0.31 & $-0.45 ;-0.16$ & $<.001$ \\
& General health condition** & 17.99 & $11.44 ; 24.53$ & $<.001$ \\
\hline
\end{tabular}

$*$ Reference category $=$ not feeling that something should change in the work situation

$* *$ Reference category $=$ being in good health
In the secondary regression analysis (Table 5), the four predictors explained $43.1 \%$ of the variance $(p<0.01)$ of the self-perceived listening effort. Feeling that something should change at work, BHI, auditory work demands, and the personal adaptations scale score were significantly related to self-perceived listening effort. In both analyses, there were no significant interaction effects.

\section{Discussion}

The aim of this study was to identify factors influencing NFR and listening effort in hearing-impaired employees. Four factors were shown to directly influence NFR and four factors were shown to directly influence self-perceived listening effort.

In line with the literature arguing the theoretical assumption that increased listening effort may cause a sense of mental fatigue (McGarrigle et al. 2014), self-perceived listening effort was found to be the factor with the highest association with NFR $(r=0.527)$ in the correlation analysis. In contrast, no significant associations were observed between the hearing test outcomes and NFR, including BHI, maximum discrimination score, and the critical SNR. While tinnitus has earlier been shown to be associated with NFR (Juul Jensen et al. 2018), we did not find a significant association in this study. This may be explained by the dichotomous question that we used that did not allow for differentiating in degree of tinnitus. Also, because we used routinely obtained healthcare data, we may have missed hearing-related factors, such as hyperacusis. For concepts related to NFR, mixed results are presented for hearing loss (Hornsby and Kipp 2016; Svinndal et al. 2018). Tone audiometry was not significantly related to fatigue and vigor (Hornsby and Kipp 2016), but patients with more severe hearing loss reported lower workability and higher degrees of fatigue (Svinndal et al. 2018).

The lack of a significant association between SNR and NFR in the correlation analysis contrasts the results of an earlier study that found poorer SNR to be associated with higher NFR (Nachtegaal et al. 2009). In this earlier study, SNR was derived from an adaptive digits-in-noise test performed over the internet and the subjects completed the test without hearing aids. In our study, routinely healthcare data
Table 5 Results of multiple linear regression analysis of factors associated with selfperceived listening effort

\begin{tabular}{llrlr}
\hline & Variable & \multicolumn{1}{l}{ B } & $95 \%$ CI & $p$ \\
\hline Complete case & Constant & 6.78 & $3.77 ; 9.80$ & $<0.001$ \\
analysis & Feeling something should change* & 1.96 & $1.12 ; 2.80$ & $<0.001$ \\
$R^{2}=.0408$ & Binaural hearing loss & 0.01 & $0.01 ; 0.02$ & $<0.001$ \\
& Auditory work demands & 0.19 & $0.12 ; 0.26$ & $<0.001$ \\
& Personal adaptations & -0.05 & $-0.06 ;-0.03$ & $<0.001$ \\
Pooled analysis & Constant & 6.10 & $3.35 ; 8.84$ & $<0.001$ \\
after imputation & Feeling something should change* & 1.82 & $1.03 ; 2.61$ & $<0.001$ \\
$R^{2}=0.431$ & Binaural hearing impairment & 0.01 & $0.01 ; 0.02$ & $<0.001$ \\
& Auditory work demands & 0.20 & $0.14 ; 0.26$ & $<0.001$ \\
& Personal adaptations & -0.05 & $-0.06 ;-0.03$ & $<0.001$ \\
\hline
\end{tabular}

$*$ Reference category $=$ not feeling that something should change in the work situation 
were used, having the advantage that all hearing tests were performed in a standardized audio cabins, but with the disadvantage that SNR data were missing in 158 patients (54\%). Performing the speech reception test in noise is not obligatory in standard care. The choice to perform the speech reception test is determined by a patient's profession and associated auditory demands. Therefore, the missing SNR data are not missing at random, and the presence of confounding cannot be ruled out. Another explanation might be that we derived SNR's with and without hearing aids, to resemble patients' daily life work situation. Although we expected the SNR scores to be more strongly associated with NFR, this choice may have masked an existing association.

Since BHI correlates with self-perceived listening effort, but not with NFR, we presume that the degree of hearing loss is not the underlying factor explaining the moderate correlation between listening effort and NFR. In the directed acyclic graph, two factors show moderate correlations with both self-perceived listening effort and NFR, specifically: feeling that something should change at work and personal adaptations. Apparently, the way employees perceive their hearing difficulties and how they cope with their hearing loss influence their listening effort and the fatigue experienced after a day of work. Likewise, subjective measures of perceived hearing difficulties were found to be strongly associated with fatigue and vigor, whereas there was no significant association with degree of hearing loss (Hornsby and Kipp 2016). It would be interesting to compare our findings of self-reported listening effort with other measures of listening effort, such as measuring reaction time or pupil responses during speech reception tasks in noise (McGarrigle et al. 2014).

In line with de Vries et al. (2015) and Machin and Hoare (2008), we found a significant correlation between coping behavior and NFR. Specifically, we have explored two variables for coping behavior distinguishing the communication strategies that were used and the personal adaptations that were made. Although these scores showed a moderate correlation between themselves $(r=0.399)$, the personal adaptation score was directly related to self-perceived listening effort and NFR, but the communication strategy score was not. Other studies report an association between communication strategies used and NFR (de Vries et al. 2015; Machin and Hoare 2008). Having a passive reaction coping style explained $26 \%$ of the variance in NFR in employees with major depression in remission (de Vries et al. 2015). In a population of bus drivers, maladaptive driver coping behaviors were shown to be associated with NFR (Machin and Hoare 2008). To our knowledge, previous studies have not focused on the association between NFR and personal adaptations, including self-acceptance, acceptance of loss, and stress and withdrawal. A qualitative study reported that self-acceptance facilitates work ability (Detaille et al. 2003).
Distinguishing communication strategies and personal adaptations would be of interest in future studies with hearingimpaired employees to gain further understanding of the influence of coping behavior on NFR.

In addition to explore the influence of coping behavior, several other questions were included to assess the influence of personal factors. We observed that the factor feeling that something should change at work was moderately associated with NFR, as well as with self-perceived listening effort. The question "Do you feel something should change in your work situation" may grasp a feeling of frustration at the workplace, that was earlier associated with NFR in seafarers (Bridger et al. 2010). Feeling frustration at the workplace might be associated with higher need for recovery. This finding must; however, be interpreted with caution, because we used a single question, rather than a validated questionnaire. The question may also reflect other constructs, such as the awareness or acceptance of functional hearing difficulties at the workplace. Although a firm conclusion can thus not yet been drawn, this finding underlines the importance to measure employees' frustration level in future research concerning NFR using a validated questionnaire.

In line with the previous studies (Gommans et al. 2015; van der Starre et al. 2013), general health condition was found to be significantly associated with NFR, independently from the other factors. Age, gender, and educational level were considered to be potential confounders, but the correlation analysis showed that these factors were neither significantly associated with NFR, nor with hearing related or personal factors. A similar independent position was found for the factor auditory demands. This factor was moderately associated with self-perceived listening effort. In contrast to the literature describing that auditory demands are significantly related to hearing handicap and sick leave (Kramer et al. 1998, 2006), we did not find a significant association between auditory demands at work and NFR. This may suggest that although high auditory demands increase the listening effort, the degree of feeling fatigued after work depends on other factors. The use of personal adaptations or being in good health may be protective for developing occupational problems. Future research is required to further assess these mechanisms.

Other work characteristics did neither influence NFR nor self-perceived listening effort. First, we expected a positive association between the number of working hours and NFR (Jansen et al. 2002; Verdonk et al. 2010), but this was not the case. The lack of association could be explained if patients with high NFR had chosen to work fewer hours to prevent health problems. Since this study uses health administrative data, we cannot confirm this hypothesis. The directed acyclic graph showed that men had a higher number of working hours than women, which is a typical finding for the Dutch working population 
(Gjerdingen et al. 2001). Employees that reported a higher number of working hours, more often reported fulfilling managerial tasks and those fulfilling managerial tasks reported being more able to participate in work decisions. Second, in contrast to what was observed earlier (van Veldhoven and Broersen 2003), work participation was not associated with NFR. In other words, the feeling of job control did not directly influence NFR. Literature presents mixed results on the association between job control and NFR (Kraaijeveld et al. 2014; Sonnentag and Zijlstra 2006; Van der Hulst et al. 2006). Third, receiving collegial support did neither influence the feeling that something should change at work, nor NFR as was earlier reported (Kraaijeveld et al. 2014). This might be explained by the small variance in collegial support reported by our population. Only a few employees reported having problems in their relationship with colleagues.

We have derived work-related factors from the QEEW and $\mathrm{ACHW}$, because these questionnaires are routinely performed in the ENT-Audiology clinic. Therefore, we may have missed other work-related factors that influence NFR in hearing-impaired employees, such as job control, job demand, and social support. The included scale score of collegial support does not reflect all aspects of the construct social support, since this construct also refers to helpful social interactions from supervisors (Nachtegaal et al. 2009). For future research, we recommend to include the Job Content Questionnaire when measuring psychosocial work characteristics (Karasek et al. 1998).

Some study limitations should be noted. First, the retrospective character of the study implicates a risk for measurement bias. For example, the hearing tests were performed by multiple clinicians following clinical protocols, rather than a research protocol, which may have caused differences in measurement settings. Despite this limitation, the four identified factors accounted for $46.1 \%$ of the variance in NFR and $43.1 \%$ of self-perceived listening effort. Second, the cross-sectional design is a limitation of this study, since it does not allow drawing conclusions about causality. Constructing a directed acyclic graph allowed for visualization of the relationship of a broad spectrum of factors influencing NFR. Since the evidence on factors influencing NFR in hearing-impaired employees was limited, this explorative method is considered to be appropriate. A prospective study is needed to verify and validate the findings of this study. To gain further understanding in the difficulties of hearing-impaired employees and the efficacy of intervention strategies that aim to reduce these difficulties, future clinical trials are recommended to assess the efficacy of audiological, speech therapeutic, and social interventions on both self-perceived listening effort and NFR.

\section{Concluding remarks}

This study provides a framework of factors associated with NFR in hearing-impaired employees, contributing to the understanding of occupational problems in this population. The results suggest that the way employees perceive their hearing loss and how they cope with it directly influence NFR, rather than their measured degree of hearing loss. Further, when assessing or evaluating NFR, an employees' general health condition should be considered. These findings are relevant for clinicians and occupational physicians that perform diagnostics or intervention strategies for hearing-impaired employees. Also, the results may contribute to gain understanding in the working mechanisms of interventions that aim to prevent or cure occupational diseases in employees with hearing loss.

Acknowledgements The authors would like to thank Dr. R. Holman for the support with statistical analysis, Dr. I. Jacobi for providing feedback on the manuscript, and J. Lebouille, B.A. for her assistance in the data entry.

Funding No funding was received for this study.

\section{Compliance with ethical standards}

conflicts of interest The authors declare no conflicts of interest.

Ethical standards This study was conducted in accordance with the 1964 Declaration of Helsinki and its later amendments or comparable ethical standards. The medical ethics committee at the Amsterdam UMC declared that no formal approval of the detailed protocol was needed according to the Dutch Medical Research Involving Human Subjects Act (No. 18_369 \# 18.421).

Open Access This article is licensed under a Creative Commons Attribution 4.0 International License, which permits use, sharing, adaptation, distribution and reproduction in any medium or format, as long as you give appropriate credit to the original author(s) and the source, provide a link to the Creative Commons licence, and indicate if changes were made. The images or other third party material in this article are included in the article's Creative Commons licence, unless indicated otherwise in a credit line to the material. If material is not included in the article's Creative Commons licence and your intended use is not permitted by statutory regulation or exceeds the permitted use, you will need to obtain permission directly from the copyright holder. To view a copy of this licence, visit http://creativecommons.org/licenses/by/4.0/.

\section{References}

Akoglu H (2018) User's guide to correlation coefficients. Turk J Emerg Med 18(3):91-93

Academy of Otolaryngology (Committee on Hearing and Equilibrium) (1979) Guide for the evaluation of hearing handicap. JAMA 241(19):2055-2059

Backenroth-Ohsako GAM, Wennberg P, Af Klinteberg B (2003) Personality and work life: a comparison between hearing-impaired 
persons and a normal-hearing population. Soc Behav Personal 31(2):191-204

Barker AB, Leighton P, Ferguson MA (2017) Coping together with hearing loss: A qualitative meta-synthesis of the psychosocial experiences of people with hearing loss and their communication partners. Int J Audiol 56(5):297-305

Bosman AJ, Smoorenburg GF (1995) Intelligibility of Dutch CVC syllables and sentences for listeners with normal hearing and with three types of hearing impairment. Audiology 34(5):260-284

Bridger R, Brasher K, Dew A (2010) Work demands and need for recovery from work in ageing seafarers. Ergonomics 53(8):1006-1015

Broersen JP, Fortuin RJ, Dijkstra L, van Veldhoven M, Prins J (2004) Monitor Arboconvenanten: kengetallen en grenswaarden. TBV-Tijdschrift voor Bedrijfs-en Verzekeringsgeneeskunde 12(4):104-8

Castor EDC (2019) Castor Electronic Data Capture. https://castoredc. com. Accessed 4 Feb 2019

Danermark B, Gellerstedt LC (2004) Psychosocial work environment, hearing impairment and health. Int J Audiol 43(7):383-389

de Croon EM, Sluiter JK, Frings-Dresen MH (2003) Need for recovery after work predicts sickness absence: a 2-year prospective cohort study in truck drivers. J Psychosom Res 55(4):331-339

Detaille SI, Haafkens JA, Van Dijk F (2003) What employees with rheumatoid arthritis, diabetes mellitus and hearing loss need to cope at work. Scand J Work Environ Health 29(2):134-142

de Vries G, Koeter MW, Nieuwenhuijsen K, Hees HL, Schene AH (2015) Predictors of impaired work functioning in employees with major depression in remission. J Affect Disord 185:180-187

Dobie RA (2011) The AMA method of estimation of hearing disability: a validation study. Ear Hear 32(6):732-740

Gjerdingen D, McGovern P, Bekker M, Lundberg U, Willemsen T (2001) Women's work roles and their impact on health, wellbeing, and career: comparisons between the United States, Sweden, and The Netherlands. Women Health 31(4):1-20

Gommans F, Jansen N, Stynen D, De Grip A, Kant I (2015) Need for recovery across work careers: the impact of work, health and personal characteristics. Int Arch Occup Environ Health 88(3):281-295

Greenland S, Pearl J, Robins JM (1999) Causal diagrams for epidemiologic research. Epidemiology 10:37-48

Gussenhoven AH, Anema JR, Goverts ST, Bosmans JE, Festen JM, Kramer SE (2012) Cost-effectiveness of a vocational enablement protocol for employees with hearing impairment; design of a randomized controlled trial. BMC Public Health 12:151

Gussenhoven AHM, Anema JR, Witte BI, Goverts ST, Kramer SE (2017) The Effectiveness of a vocational enablement protocol for employees with hearing difficulties: results of a randomized controlled trial. Trends Hear 21:1-12

Hallberg LR-M, Carlsson SG (1991) A qualitative study of strategies for managing a hearing impairment. Br J Audiol 25(3):201-211

Hasson D, Theorell T, Wallén MB, Leineweber C, Canlon B (2011) Stress and prevalence of hearing problems in the Swedish working population. BMC public health 11(1):130

Hasson D, Theorell T, Westerlund H, Canlon B (2010) Prevalence and characteristics of hearing problems in a working and nonworking Swedish population. J Epidemiol Community Health 64(5):453-460

Hinkle DE, Wiersma W, Jurs SG (2003) Applied statistics for the behavioral sciences. Houghton Mifflin College Division, Boston

Hornsby BW, Kipp AM (2016) Subjective Ratings of Fatigue and Vigor in Adults with Hearing Loss Are Driven by Perceived Hearing Difficulties Not Degree of Hearing Loss. Ear Hear 37(1):e1-10
Jansen NW, Kant I, van den Brandt PA (2002) Need for recovery in the working population: description and associations with fatigue and psychological distress. Int J Beh Med 9(4):322

Juul Jensen J, Callaway SL, Lunner T, Wendt D (2018) Measuring the impact of tinnitus on aided listening effort using pupillary response. Trends Hear 22:1-17

Karasek R, Brisson C, Kawakami N, Houtman I, Bongers P (1998) The job content questionnaire (JCQ): An instrument for internationally comparative assessments of psychosocial job characteristics. J Occ Health Psychol 3:322-355

Kiss P, De Meester M, Braeckman L (2008) Differences between younger and older workers in the need for recovery after work. Int Arch Occup Environ Health 81(3):311-320

Kraaijeveld RA, Huysmans MA, Hoozemans MJ, Van der Beek AJ, Speklé EM (2014) The influence of psychosocial work characteristics on the need for recovery from work: a prospective study among computer workers. Int Arch Occup Environ Health $87(3): 241-248$

Kraemer HC (2014) Biserial correlation. Wiley StatsRef: Statistics Reference Online

Kramer SE, Kapteyn TS, Festen JM, Kramer SE (1998) The selfreported handicapping effect of hearing disabilities. Audiology 37(5):302-312

Kramer SE, Kapteyn TS, Houtgast T (2006) Occupational performance: comparing normally-hearing and hearing-impaired employees using the Amsterdam Checklist for Hearing and Work. Int J Audiol 45(9):503-512

Machin MA, Hoare PN (2008) The role of workload and driver coping styles in predicting bus drivers' need for recovery, positive and negative affect, and physical symptoms. Anxiety Stress Coping 21(4):359-375

Masterson EA, Themann CL, Luckhaupt SE, Li J, Calvert GM (2016) Hearing difficulty and tinnitus among US workers and nonworkers in 2007. Am J Ind Med 59(4):290-300

May JJ (2000) Occupational hearing loss. Am J Ind Med 37(1):112-120

Martin FN, Champlin CA (2000) Reconsidering the limits of normal hearing. J Am Acad Audiol 11(2):64-66

McGarrigle R et al (2014) Listening effort and fatigue: What exactly are we measuring? A British society of audiology cognition in hearing special interest group 'white paper'. Int J audiol 53(7):433-440

Mokkink LB, Knol DL, Zekveld AA, Goverts ST, Kramer SE (2009) Factor Structure and Reliability of the Dutch Version of Seven Scales of the Communication Profile for the Hearing Impaired (CPHI). J Speech Lang Hear Res 52:454-464

Morata TC, Themann CL, Randolph RF, Verbsky BL, Byrne DC, Reeves ER (2005) Working in noise with a hearing loss: perceptions from workers, supervisors, and hearing conservation program managers. Ear Hear 26(6):529-545

Moriguchi CS, Alem MER, Mv V, Coury HJCG (2010) Cultural adaptation and psychometric properties of Brazilian need for recovery scale. Rev Saude Publica 44:131-139

Nachtegaal J, Kuik DJ, Anema JR, Goverts ST, Festen JM, Kramer SE (2009) Hearing status, need for recovery after work, and psychosocial work characteristics: results from an internet-based national survey on hearing. Int J Audiol 48(10):684-691

Nelson DI, Nelson RY, Concha-Barrientos M, Fingerhut M (2005) The global burden of occupational noise-induced hearing loss. Am J Ind Med 48(6):446-458

Ohlenforst B et al (2017) Effects of hearing impairment and hearing aid amplification on listening effort: A systematic review. Ear Hear 38(3):267-281

World Health Organization (2001) International classification of functioning, disability and health: ICF. World Health Organization, Geneva 
Pedersen AB et al (2017) Missing data and multiple imputation in clinical epidemiological research. Clin Epidemiol 9:157-166

Plomp R, Mimpen A (1979) Improving the reliability of testing the speech reception threshold for sentences. Audiology 18(1):43-52

Punch R (2016) Employment and Adults Who Are Deaf or Hard of Hearing: Current Status and Experiences of Barriers, Accommodations, and Stress in the Workplace. Am Ann Deaf 161(3):384-397

Sherbecoe RL, Studebaker GA (2004) Supplementary formulas and tables for calculating and interconverting speech recognition scores in transformed arcsine units. Int J Audiol 43(8):442-448

Shrier I, Platt RW (2008) Reducing bias through directed acyclic graphs. BMC Med Res Methodol 8(1):70

Sluiter JK (1999) The influence of work characteristics on the need for recovery and experienced health: a study on coach drivers. Ergonomics 42(4):573-583

Sluiter J, De Croon E, Meijman T, Frings-Dresen M (2003) Need for recovery from work related fatigue and its role in the development and prediction of subjective health complaints. Occup Environ Med 60(suppl 1):i62-i70

Sluiter JK, Frings-Dresen MH, van der Beek AJ, Meijman TF (2001) The relation between work-induced neuroendocrine reactivity and recovery, subjective need for recovery, and health status. J Psychosom Res 50(1):29-37

Smits C, Merkus P, Houtgast T (2006) How we do it: The Dutch functional hearing-screening tests by telephone and Internet. Clin Otolaryngol 31(5):436-440

Sonnentag S, Zijlstra FR (2006) Job characteristics and off-job activities as predictors of need for recovery, well-being, and fatigue. $\mathrm{J}$ Appl Psychol 91(2):330-350

Sorgdrager B (2015) Gehoorverlies in het werk: over lawaaischade en functioneringsproblemen. TBV-Tijdschrift voor Bedrijfs-en Verzekeringsgeneeskunde 23(7):320-3

Svinndal EV, Solheim J, Rise MB, Jensen C (2018) Hearing loss and work participation: a cross-sectional study in Norway. Int $\mathrm{J}$ Audiol 57(9):646-656
Van der Hulst M, Van Veldhoven M, Beckers D (2006) Overtime and need for recovery in relation to job demands and job control. $\mathrm{J}$ Occup Health 48(1):11-19

van der Starre RE, Coffeng JK, Hendriksen IJ, van Mechelen W, Boot CR (2013) Associations between overweight, obesity, health measures and need for recovery in office employees: a cross-sectional analysis. BMC Public Health 13(1):1207-1220

van Veldhoven M, Broersen S (2003) Measurement quality and validity of the "need for recovery scale". Occup Environ Med 60(suppl 1): $\mathrm{i} 3-\mathrm{i} 9$

Van Veldhoven M, Meijman T, Broersen J (2002) Fortuin R (2002) Handleiding VBBA. SKB Vragenlijst Services, Amsterdam

van Veldhoven MJ, Sluiter JK (2009) Work-related recovery opportunities: testing scale properties and validity in relation to health. Int Arch Occup Environ Health 82(9):1065-1075

van Veldhoven M, Prins J, van der Laken P, Dijkstra L (2015) QEEW2. 0: 42 short scales for survey research on work, well-being and performance.

Verdonk P, Hooftman WE, van Veldhoven MJ, Boelens LR, Koppes LL (2010) Work-related fatigue: the specific case of highly educated women in the Netherlands. Int Arch Occup Environ Health 83(3):309-321

Versfeld NJ, Daalder L, Festen JM, Houtgast T (2000) Method for the selection of sentence materials for efficient measurement of the speech reception threshold. J Acoust Soc Am 107(3):1671-1684

Whitley R (1989) On the nature of managerial tasks and skills: their distinguishing characteristics and organization. J Manag Stud 26(3):209-224

Publisher's Note Springer Nature remains neutral with regard to jurisdictional claims in published maps and institutional affiliations. 